\section{PSICOLOGIA IBEROAMERICANA}

\section{Psicología lberoamericana}

ISSN: 1405-0943

psicología.iberoamericana@uia.mx

Universidad Iberoamericana, Ciudad de

México

México

Guzmán Saldaña, Rebeca María Elena; Gómez Peresmitré, Gilda; García Meraz, Melissa; del Castillo Arreola, Arturo

Análisis Factorial Confirmatorio del Inventario de Autoeficacia Percibida para Control de Peso en Población Mexicana

Psicología lberoamericana, vol. 19, núm. 2, julio-diciembre, 2011, pp. 78-88

Universidad Iberoamericana, Ciudad de México

Distrito Federal, México

Disponible en: http://www.redalyc.org/articulo.oa?id=133921440009

Cómo citar el artículo

Número completo

- Más información del artículo

Página de la revista en redalyc.org

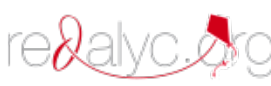

Sistema de Información Científica

Red de Revistas Científicas de América Latina, el Caribe, España y Portugal Proyecto académico sin fines de lucro, desarrollado bajo la iniciativa de acceso abierto 


\title{
Análisis Factorial Confirmatorio del Inventario de Autoeficacia Percibida para Control de Peso en Población Mexicana
}

\author{
Factorial Confirmatory Analysis of the Inventory of Perceived \\ Self Efficacy for Weight Control in Mexican Population
}

\author{
Rebeca María Elena Guzmán Saldaña \\ Gilda Gómez Peresmitré \\ Universidad Autónoma de México, CD. de México* \\ Melissa García Meraz \\ Arturo del Castillo Arreola \\ Universidad Autónoma del Estado de Hidalgo, México**
}

\section{RESUMEN}

El propósito de este estudio fue adaptar, validar y confiabilizar el Inventario de Autoeficacia Percibida para Control de Peso (AP-CP) en población escolar mexicana. La muestra no probabilística quedó formada por $N=831$ estudiantes con $M_{\text {edad }}=12,(D E=1.70)$, de escuelas públicas de Pachuca, Hidalgo, México. Para efecto de tal propósito se aplicaron análisis factoriales exploratorios y confirmatorios. Se obtuvieron dos versiones, una para hombres y otra para mujeres, del AP-CP. El análisis confirmatorio arrojó dos modelos de tres factores cada uno, con un buen ajuste de los datos y consistencia interna aceptable. Se encontró que la autoeficacia percibida para control de peso y actividad física se relaciona con el género; para control del peso los hombres señalan a la fuerza de voluntad, actividad física e ingesta alimentaria; las mujeres hacen referencia a conducta alimentaria y a actividad física programada y cotidiana. Se discute la utilidad del Inventario AP-CP para la prevención de la obesidad en las primeras etapas de vida.

Palabras clave: Autoeficacia; control de peso; análisis factorial confirmatorio.

\section{ABSTRACT}

The present study tested the adaptation, reliability and validity of the Inventory of the Perceived Self Efficacy for the Weight Control. The non-random sample was comprised of $N=831$ Mexican students, $\left(M_{a g e}=12, S D=1.70\right)$ of public schools in Pachuca, Hidalgo, Mexico. The confirmatory factorial produced two models; three factors each, with good fit and good reliability. The results show that the perceived self efficacy for weight control and physical activity is related to gender: for weight control, men makes reference to will force, physical activity and eating behavior; while for women control $r$ weight is related to eating behavior, and to programmed and daily physical activity

The discussion of this it can be useful in prevention of obesity in life first stages. Finally it is discussed the usefulness of the Inventory of the Perceived Self Efficacy in prevention is discussed.

Key words: Self efficacy; weight control; factorial confirmatory analysis

\footnotetext{
* Para correspondencia: Facultad de Psicología de la Universidad Nacional Autónoma de México, Cubículo 11, Mezzanine, Av. Universidad 3004, Col. Copilco-Universidad, Delegación Coyoacán, 04510 México, D.F. Tel. (55)56-22-22-52, fax (55)56-16-07-78. Correos electrónicos: remar64@yahoo. com.mx; gildag@servidor.unam.mx

** Para correspondencia: Universidad Autónoma del Estado de Hidalgo, Ex hacienda La Concepción carretera Pachuca-Actopan, Tel. 04455 20257684, correos electrónicos: melissaunam@yahoo.com.mx; artur78@yahoo.com
} 


\section{INTRODUCCIÓN}

La actividad física (AF) como componente de un estilo de vida saludable disminuye el riesgo de padecer enfermedad coronaria (Batty \& Lee, 2004), hipertensión arterial, obesidad y diabetes (American Diabetes Association, 2010); además, favorece el bienestar emocional (Pianosi \& Davis, 2004; Salmon \& Timperio, 2007). Contrariamente, el sedentarismo incrementa el riesgo de adquirir enfermedades crónicas no transmisibles, y en los niños y adolescentes eleva la probabilidad de sufrir enfermedades de adultos, como la diabetes tipo 2 (Daniels \& Greer, 2008; Juarbe, Lipson \& Turok, 2003).

La AF facilita el movimiento de los músculos esqueléticos y eleva el gasto energético por encima del metabolismo basal (Weinberg, 1966). Según Dunn, Marcus, Kampert, Garcia, Kohl \& Blair (1999), la AF tiene dos dimensiones: a) cotidiana: acciones de la vida diaria como andar, subir escaleras, hacer tareas del hogar, y $b$ ) programada: actividades planificadas y diseñadas para estar en forma y gozar de buena salud, como andar a paso ligero y montar en bicicleta.

Ahora bien, el control de peso (CP) incluye los hábitos alimenticios en los que influyen estímulos externos (que controlan la ingesta como la hora del día, el aspecto, el sabor y olor de la comida) (Román, Díaz, Cárdenas \& Lugli, 2007; Saldaña, 1994; Saldaña \& Rossell, 1988).

En el CP, factores psicológicos como los procesos volitivos cobran gran relevancia; éstos son definidos como estrategias de control cognoscitivo, emocional, motivacional y medioambiental, que se observan en personas orientadas hacia la acción (Kuhl, 1994). Flórez-Alarcón (2007) señala que en el proceso de modificación del peso corporal, estos procesos establecen patrones comportamentales que intervienen en el $\mathrm{CP}$; facilitando el establecimiento de contingencias situacionales específicas (cómo, cuándo y dónde del comportamiento de ingesta de alimentos y de $\mathrm{AF}$ ), además de soluciones alternativas para acceder a un comportamiento favorable al CP (como caminar diariamente). Así, es más probable que la persona maneje adecuadamente las situaciones asociadas con los comportamientos de ingesta alimentaria y AF, cuyo objetivo es el CP.

Una serie de factores psicológicos, demográficos y del medio ambiente físico y social están relacionados con la AF y el CP (Cabrera, Gómez \& Mateus, 2004). Entre los psicológicos destaca la autoeficacia como uno de los más relevantes (Márquez \& MacAuley, 2006; Ryan 2005). Según Bandura (1998), ésta es la convicción de tener éxito al llevar a cabo una conducta, ya sea cambiándola o manteniéndola. La personas con alta autoeficacia son optimistas y realizan cuidados preventivos; además, se enferman y deprimen menos (Flores León, González-Celis Rangel \& Valencia Ortiz, 2010; Vinaccia, Contreras, Restrepo, Cadena \& Anaya, 2005).

La autoeficacia predice el logro de acciones ligadas a tratamientos para el CP y su mantenimiento (Astudillo García \& Rojas Russel, 2006; Jago, Baranowski, Baranowski, Cullen \& Thompson, 2007); por ejemplo, se relaciona con la intención de comer alimentos bajos en calorías, frutas y vegetales (Povey, Conner, Sparks, James \& Shepherd, 2000).

Para evaluar el concepto de autoeficacia se han creado diversos instrumentos. A nivel internacional se cuenta con las escalas: Autoeficacia General (General Self-Efficacy Scale) de Schwarzer y Jerusalem, (1995), Regularidad del Ejercicio (Exercise Regularly Scale) de Lorig, Stewart, Ritter, Gonzalez, Laurent \& Lynch (1996), Autoeficacia en Nutrición (The Nutrition Self-Efficacy Scale) de Schwarzer y Renner, (2000),y Anderson, Winett y Wojcik, (2007), y Autoeficacia para Ejercicio (Exercise Self-Efficacy Scale) de Schwarzer y Renner (2000)Para evaluar la conducta alimentaria y autoeficacia se diseñó la Escala de Autoeficacia (SelfEfficacy Scale) de Glynn y Ruderman (1986), y el Cuestionario de Estilo de Vida, Autoeficacia y Peso (Weight Efficacy Life-Style Questionnaire) de Clark, Abrams, Niaura, Eaton y Rossi (1991), adaptados al castellano por Ruiz, Berrocal, López y Rivas (2002, 2003).

En México, dos escalas que evalúan la autoeficacia, específicamente en niños son: Autoeficacia hacia la Actividad Física en Niños de Edad Escolar (Aedo \& Ávila, 2009), y Autoeficacia para Realizar Conductas Saludables en Niños Sanos (Flores León, GonzálezCelis Rangel \& Valencia Ortiz, 2010). La primera identifica tres factores: búsqueda de alternativas positivas hacia la AF, capacidad para enfrentar posibles barreras para llevarla a cabo y expectativas de habilidad o competencia. La segunda mide actividades de protección a la salud y actividades de independencia. 
A pesar de los avances en cuanto a la medición del constructo de autoeficacia, Stice, Shaw y Marti (2006) destacan la necesidad de desarrollar más estudios sobre la medida y estimación de ésta para CP y AF, dado que son pocos los instrumentos que se han elaborado para tal fin, particularmente en población infantil y juvenil.

Por ello, el objetivo del presente estudio fue adaptar, validar y confiabilizar, en población escolar mexicana, el Inventario de Autoeficacia Percibida para Control de Peso (Román et al., 2007).

\section{MÉTODO}

\section{Participantes}

La muestra no probabilística se conformó por $N=831$ participantes, entre 10 y 16 años $\left(M_{\text {edad }}=12, D E=1.70\right)$, estudiantes de escuelas públicas del nivel básico, de Pachuca, Hidalgo, México. 51\% $(n=421)$ fueron mujeres y $49 \%(n=410)$ hombres.

\section{Instrumento}

Inventario de Autoeficacia Percibida para Control de Peso (AP-CP). Su versión original se desarrolló en población escolar venezolana, es una sola versión para ambos sexos. Consta de 37 ítems tipo Likert, con cuatro opciones de respuesta ("incapaz de poder hacerlo" hasta "seguro de poder hacerlo"). Con el método de los Componentes Principales con rotación VARIMAX se obtuvieron tres factores: estilo de alimentación y externalidad con 20 ítems (alpha $=.91$ ), actividad física programada con nueve (alpha $=.88$ ), y actividad física cotidiana con ocho (alpha $=.88$ ); que en su conjunto, explican $44.08 \%$ de la varianza total. Para mayor información, ver Román y cols. (2007).

De acuerdo con el objetivo de esta investigación, el AP-CP original se modificó después de realizar un estudio piloto en donde se aplicó a 100 estudiantes de educación básica (con características sociodemográficas similares a la muestra de investigación); los cambios principales consideraron los hábitos, la edad y el contexto socio-cultural de los jóvenes mexicanos; además, se ajustó la equivalencia semántica de cada uno de los ítems. Así, se integró una versión final del AP-CP con un total de 45 ítems. A los 37 ítems originales se le agregaron ocho más, cuatro que incluyeron información sobre variables sociodemográficas y cua- tro sobre procesos volitivos relacionados con la realización de $\mathrm{AF}$ y $\mathrm{CP}$; se mantuvo el mismo formato tipo Likert con cuatro opciones de respuesta, que van desde "No puedo hacerlo" (1) hasta "Seguro que puedo hacerlo" (4). Una puntuación alta se interpreta como alta autoeficacia.

\section{Procedimiento}

La versión modificada del AP-CP fue aplicada por estudiantes de psicología previamente capacitados, de forma grupal en las aulas escolares, dentro del horario de clases. El tiempo de aplicación fue de 15 a 25 minutos. Con la finalidad de resguardar aspectos éticos, se solicitó previamente el consentimiento informado tanto de los padres, como de los participantes.

Análisis estadísticos. Antes de efectuar el análisis confirmatorio, se desarrolló un análisis factorial exploratorio debido a que previamente no se contaba con datos del Inventario AP-CP en población mexicana. Éste se llevó a cabo con el método de los Componentes Principales con rotación VARIMAX (SPSS versión 17.0); posteriormente, se realizó el análisis factorial confirmatorio de los datos obtenidos en el exploratorio, mediante el programa de Ecuaciones Estructurales AMOS (versión 12.0), probándose modelos para hombres y para mujeres.

Para evidenciar la medida en que los modelos reproducían las relaciones en la matriz de correlaciones de los datos empíricos, los modelos hipotetizados se evaluaron con el grado de bondad de ajuste global, considerando los siguientes índices: el valor de Chi Cuadrada no significativo, el Error Cuadrático Medio de Aproximación por Grados de Libertad (RMSEA) con un valor pequeño $(<.05)$ que representa un buen ajuste; los índices GFI y corregido, según los grados de libertad AGFI, con valores de .90 hasta .95 indican un ajuste adecuado, y por arriba de .95 un ajuste bueno (Bollen \& Long, 1993).

Es importante señalar que con anticipación se aplicó la prueba $t$ de Student para comparar respuestas por sexo para cada uno de los ítems, las diferencias estadísticamente significativas de los mismos fundamentaron la obtención de dos versiones del instrumento, una para hombres y otra para mujeres.

Como primer paso, se evaluó la adecuación de la matriz de correlaciones, utilizando el índice de Kaiser- 
Meyer-Olkin, obteniendo un valor satisfactorio para ambas versiones. El test de Esfericidad de Barlett fue significativo, lo que indicó correlaciones adecuadas y la pertinencia para realizar el análisis factorial. Para éste se consideraron los ítems que obtuvieron una carga factorial $\geq .40$, y se aceptaron como factores solamente los que contenían como mínimo tres ítems (Hair, Anderson, Tatham \& Blakc, 1999).

\section{RESULTADOS}

\section{Inventario de Hombres}

Análisis Factorial Exploratorio. La aplicación del método de los Componentes Principales con rotación VARIMAX arrojó seis factores que integraron 36 ítems, explicando $45.11 \%$ de la varianza total, después de 23 iteraciones, y un alfa de Cronbach total= 94 .

Los factores obtenidos fueron: 1) AF programada (10 ítems) con alfa $=.87$ y varianza explicada $=12.53 \%$;
2) estilos alimenticios no saludables (ocho ítems) con alfa $=.81$ y varianza explicada $=10.02 \% ; 3$ ) voluntad para controlar la ingesta alimentaria y actividad física (seis ítems) con alfa=.79 varianza explicada $=7.52 \% ; 4$ ) actividad física y fuerza de voluntad (cinco ítems) con alfa $=.77$ y varianza explicada $=6.27 \%$; 5) estilos alimenticios y externalidad (cuatro ítems) con alfa $=.65$ varianza explicada $=5.01 \%$; y 6) estilos alimenticios (tres ítems) con alfa $=.64$ varianza explicada $=3.76 \%$. Análisis Factorial Confirmatorio. Después de realizar varios ajustes a las soluciones factoriales para hombres, utilizando el método de Máxima Verosimilitud se obtuvo el modelo representado en la Figura 1, que muestra los índices de bondad de ajuste con valores de: 1) Chi cuadrado no significativo $\left(X^{2}=74.76, p=.453\right)$, con $g l=74$; 2) $A G F I=.961$; 3) $N F I=.949$, 4) $C F I=.999$, y 5) $R M S E A=.005$; todos estos puntajes indicaron un buen ajuste.

Figura 1. Modelo e índices de ajuste del AP-CP en hombres

\begin{tabular}{|c|c|}
\hline $\begin{array}{c}\text { ÍNDICES DE } \\
\text { AJUSTE }\end{array}$ & MODELO \\
\hline$X^{2}$ & 74.8 \\
\hline SIG & .453 \\
\hline$G F I$ & .974 \\
\hline$A G F I$ & .961 \\
\hline NFI & .949 \\
\hline CFI & .999 \\
\hline$R M S E A$ & .005 \\
\hline$G L$ & 74 \\
\hline
\end{tabular}

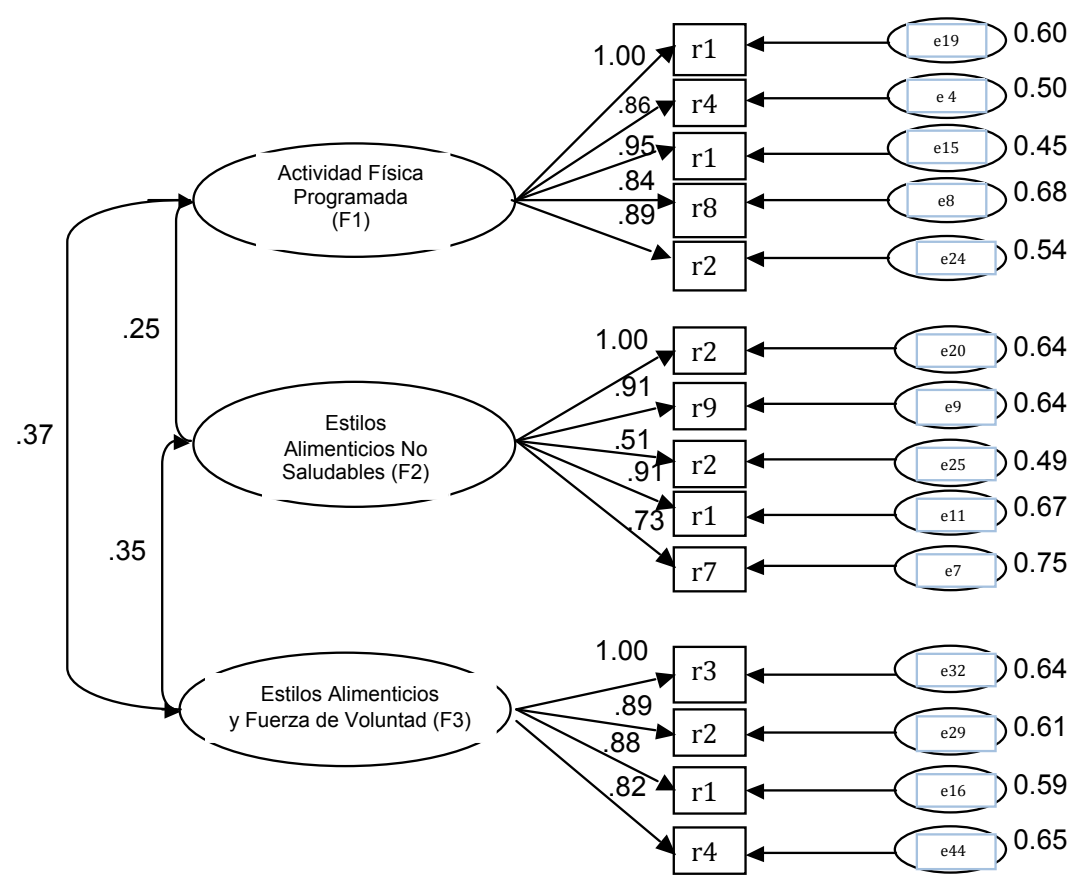


Este modelo incluyó tres variables exógenas (en óvalos grandes), además de variables endógenas (representadas con rectángulos e identificadas con el número de ítem de la variable latente a la que pertenece), y los errores de medida de las variables exógenas (e) (en óvalos pequeños). Las cargas factoriales confirmaron la solución de tres factores: 1) $A F$ programada, 2) estilos alimenticios no saludables, y 3 ) estilos alimenticios y fuerza de voluntad. En total fueron 14 ítems, con alpha de Cronbach $=.85($ ver Tabla 1$)$. (nueve ítems) con alfa $=.86$, y $12.15 \%$ de varianza explicada; 2) estilos alimenticios no saludables (ocho ítems) con alfa $=.86$, y varianza explicada $=10.8 \%$ de la varianza total; 3 ) estilos alimenticios y externalidad (seis ítems) con alfa $=.78$ y varianza explicada $=8.1 \%$; 4$) A F$ cotidiana (siete ítems) con alfa $=.80$ y varianza explicada $=9.45 \%$; 5) $A F$ programada (cinco ítems), con alfa $=.78$ y varianza $=6.75 \%$; 6 ) estilos alimenticios (cinco ítems) con alfa $=.76$ y varianza explicada $=6.75 \%$.

Tabla 1. Cargas factoriales del modelo para hombres

\begin{tabular}{|c|l|c|c|c|}
\hline No. & Ítems & F1 & F2 & F3 \\
\hline 15 & Correr todos los días & .715 & & \\
\hline 19 & Realizar abdominales o pesas en casa & .680 & & \\
\hline 4 & Realizar ejercicio diariamente & .658 & & \\
\hline 24 & $\begin{array}{l}\text { Realizar rutinas de ejercicios a pesar de que no tengas ganas } \\
\text { de hacerlas }\end{array}$ & .625 & & \\
\hline 8 & Realizar ejercicios o salir a caminar en un parque público & .622 & & \\
\hline 25 & Eliminar de tu dieta los dulces y chocolates & & .734 & \\
\hline 20 & Evitar los dulces aunque se te antojen & .694 & \\
\hline 11 & $\begin{array}{l}\text { Dejar de comer chocolates u otras golosinas que comes } \\
\text { cuando te sientes presionado }\end{array}$ & & .654 & \\
\hline 9 & Tener fuerza de voluntad para dejar de comer dulces & & .625 & .635 \\
\hline 7 & Evitar comer alimentos chatarra & & .517 & \\
\hline 32 & Dejar de cenar si te sientes presionado & & & \\
\hline 16 & Comer poco cuando estas con tus amigos (as) & & & \\
\hline 29 & Dejar de comer a cado rato aunque te sientas nervioso & & & \\
\hline 44 & Alfa de Cronbach=0.85 & & & \\
\hline & & & & \\
\hline
\end{tabular}

\section{Inventario de Mujeres}

Análisis Factorial Exploratorio. El AP-CP de mujeres quedó formado por 40 ítems, agrupados en seis factores, con alfa de Cronbach total $=.95$, que explicaron $50.53 \%$ de la varianza total, después de 12 iteraciones. Estos factores fueron: 1) $A F$ y fuerza de voluntad
Análisis Factorial Confirmatorio. Al igual que en el modelo de hombres, después de realizar varios ajustes a las soluciones factoriales para mujeres utilizando el método de Máxima Verosimilitud, se obtuvo un modelo representado en la Figura 2, en donde se observan los índices de bondad de ajuste con valores: 1) Chi 
cuadrado no significativo $\left(\mathrm{X}^{2}=50.736, \mathrm{p}=.090\right)$, con $g l=42$; 2) $A G F I=.958,3) N F I=.957,4) C F I=.997, y, 5)$ el valor de RMSEA=.016; lo que confirma un buen ajuste del modelo.

La solución del análisis confirmatorio del $A P-C P$ versión para mujeres logró un alpha $=.86$, y arrojó tres factores (con 12 ítems en total): 1) estilos alimenticios y externalidad; 2) $A F$ programada, y 3$) A F$ cotidiana (ver Tabla 2).

Figura 2. Modelo e índices de ajuste del AP-CP en mujeres

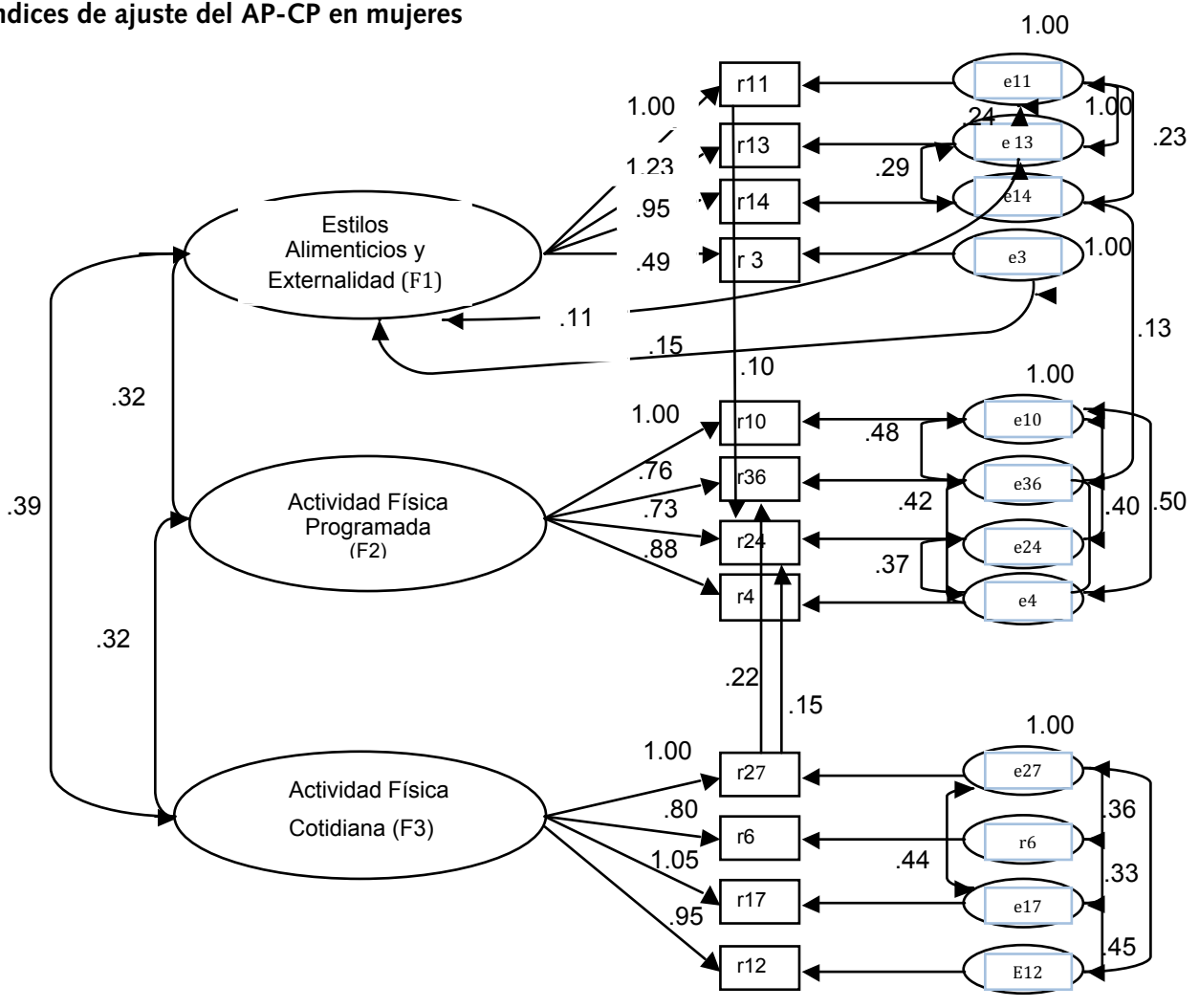

\begin{tabular}{|c|c|}
\hline ÍNDICES DE AJUSTE & MODELO \\
\hline$X^{2}$ & 54.736 \\
\hline$S I G$ & .090 \\
\hline$G F I$ & .977 \\
\hline$A G F I$ & .958 \\
\hline$N F I$ & .957 \\
\hline$C F I$ & .997 \\
\hline$R M S E A$ & .028 \\
\hline$G L$ & 42 \\
\hline
\end{tabular}


Tabla 2. Cargas factoriales del modelo para mujeres

\begin{tabular}{|c|l|c|c|c|}
\hline No. & \multicolumn{1}{|c|}{ Ítems } & F1 & F2 & F3 \\
\hline 13 & Cenar alimentos bajos en calorías & .797 & & \\
\hline 14 & Dejar de comer entre comidas con el propósito de controlar tu peso & .635 & & \\
\hline 11 & $\begin{array}{l}\text { Dejar de comer chocolates u otras golosinas que comes cuando te sientes } \\
\text { presionada }\end{array}$ & .625 & & \\
\hline 3 & Evitar ver la televisión o leer mientras comes & .398 & .696 & .644 \\
\hline 36 & Poner más esfuerzo y hacer más abdominales y pesas & & .615 & \\
\hline 24 & Realizar rutinas de ejercicios a pesar de que no tengas ganas de hacerlas & & .512 & \\
\hline 10 & Aumentar poco a poco el tiempo de duración de tu rutina de ejercicios & & .675 \\
\hline 4 & Realizar ejercicio diariamente & & .659 \\
\hline 27 & Usar las escaleras y no el elevador a pesar de estar cansada & & .597 \\
\hline 17 & Poner más esfuerzo de tu parte para subir y bajar escaleras & & .530 \\
\hline 12 & Subir y bajar escaleras en lugar de utilizar mecánicas & & \\
\hline 6 & $\begin{array}{l}\text { Caminar siempre que te sea posible evitando cualquier medio de } \\
\text { transporte }\end{array}$ & Alfa de Cronbach=0.86 & & \\
\hline
\end{tabular}

\section{DISCUSIÓN}

De acuerdo con el objetivo de este estudio, se adaptó, validó y confiabilizó exitosamente, el Inventario $A P-C P$ en población escolar mexicana (Román et al., 2007), a través de la aplicación de análisis factoriales exploratorios y confirmatorios, así como de la obtención de coeficientes de consistencia interna (alfa de Cronbrach).

En un primer momento se adaptó el Inventario, que en su versión original consta de 37 ítems, agregándose ocho más: cuatro ítems relacionados con estilos alimenticios y actividad física, vinculados con la fuerza de voluntad para controlar el peso ("dejar de comer a cada rato aunque te sientas nervioso"), y cuatro ítems sobre aspectos sociodemográficos (edad); además, se hicieron ajustes acordes con los hábitos, la edad, entre otros. En términos de la validación del Inventario, previamente se compararon las respuestas a este instrumento (a través de la prueba $t$ de Student), encontrándose diferencias significativas por sexo, y obteniendo dos versiones, una para hombres y otra para mujeres. Ambas versiones del AP-CP muestran índices totales de consistencia interna satisfactorios (.94 y .95 respectivamente).

Los análisis exploratorios arrojaron seis factores para cada versión: la de hombres está constituida por 36 ítems, y la de mujeres por 40. El porcentaje de varianza total en el Inventario de hombres explica $45.11 \%$, y en el de mujeres $50.53 \%$.

En el inventario de hombres el factor que explicó el mayor porcentaje de varianza fue actividad física programada (con 10 ítems, alfa $=.87$ y varianza=12.53); $\mathrm{y}$ en las mujeres actividad física y fuerza de voluntad (con nueve ítems alfa $=.86$, y $12.15 \%$ de la varianza total). En ambas versiones el factor de estilos alimenticios explicó el menor porcentaje de varianza (en la de hombres con alfa $=.64$ y varianza explicada $=3.76 \%$, y en la de mujeres con alfa $=.76$ y varianza explicada $=6.75 \%$ ). Cabe recordar que la versión original del inventario (que no distingue entre sexos) tiene tres factores: estilos alimenticios y externalidad (explica $17.28 \%$ de la varianza total con 20 ítems), actividad física pro- 
gramada (explicando $14.53 \%$ de varianza total con nueve ítems), y actividad física cotidiana (con $12.28 \%$ de varianza total con ocho ítems) (Román et al., 2007). Al realizar los análisis confirmatorios en la versión de hombres se obtuvieron tres factores: actividad física programada, estilos alimenticios no saludables y estilos alimenticios y fuerza de voluntad; en la de mujeres, fueron: estilos alimenticios y externalidad, actividad física programada, y actividad física cotidiana. Cabe destacar que los factores confirmados para la versión de mujeres son los mismos que los de la versión original del AP-CP; en el caso de los hombres sólo se confirmó el factor actividad física programada respecto al instrumento original.

Esta distinción en los resultados, tanto en los análisis exploratorios como en los confirmatorios, reafirma el planteamiento de la adaptación mexicana, en cuanto a que el $A P-C P$ debe contemplar versiones acordes con el sexo. Al respecto, se observa que los hombres manifiestan autoeficacia vinculada con fuerza de voluntad para el control de peso, estilos alimenticios no saludables y actividad física programada; en cambio, las mujeres relacionan la autoeficacia diferenciando a la actividad física programada de la cotidiana; asimismo, respecto a los estilos alimenticios asociados a factores externos.

En este sentido, llama la atención la función que cumplen los estereotipos de género, reproduciéndose en la autoeficacia relativa a la actividad física y al control de peso. Así, la teoría cognitivo-social aborda la diferenciación, concibiendo el género como resultado de la interacción entre factores personales (concepciones relacionadas con éste y la actividad física), factores comportamentales (patrones de actividad física relacionados con éste) y factores del entorno (reacciones sociales hacia el comportamiento vinculado con la actividad física) (Alvariñas Villaverde, Fernández Villarino \& López Villar, 2009; Bussey \& Bandura, 1999).

Por otro lado, específicamente en esta versión del $A P-C P$, la inserción de ítems vinculados al constructo "fuerza de voluntad" (dada la relevancia que diversos estudios han evidenciado en cuanto a ese constructo y la realización de actividad física y control de peso) (Armenta González-Palenzuela, 2004; Tercedor Sánchez, 2001), los resultados corroboran lo planteado por Flórez-Alarcón (2007) en cuanto a que los procesos volitivos instituyen patrones comportamentales que intervienen en el control de peso, estableciendo el cómo, cuándo y dónde de la ingesta de alimentos y la realización de actividad física; además del planteamiento de alternativas comportamentales para el control de peso (como caminar diariamente).

Con relación a los estilos alimenticios no saludables, los hombres los asocian no sólo con la fuerza de voluntad ("dejar de comer a cada rato aunque te sientas nervioso"), sino con aspectos de restricción alimentaria como eliminar de la dieta dulces, golosinas y comida chatarra; las mujeres manifiestan de forma semejante la restricción alimentaria, además la vinculan con el consumo de alimentos bajos en calorías, con disciplina, considerando factores ambientales como "evitar ver la televisión o leer mientras comes". Estos resultados van en la misma dirección de reportes previos sobre el estudio de conductas alimentarias de riesgo, en los que se observó que los hombres y las mujeres practican insistentemente la conducta dietética, aunque los hombres lo hacen en menor medida (Acosta García, Llopis Marín, Gómez Peresmitré \& Pineda García, 2005). Esta preocupación por la comida y las dietas indica que las preferencias sociales vinculadas erróneamente con la delgadez, junto con el estilo de vida, han afectado notoriamente la conducta alimentaria, así como las actividades que giran alrededor de la comida, incluso en las primeras etapas de vida (León Hernández, GómezPeresmitré \& Platas Acevedo, 2008; Gila, Castro, Toro \& Salamero, 2004).

Por otro lado, es importante considerar que los análisis confirmatorios de la versión de mujeres, específicamente relacionados con los ítems: 11 ("dejar de comer chocolates $\mathrm{u}$ otras golosinas que comes cuando te sientes presionada") y 27 ("usar las escaleras y no el elevador a pesar de estar cansada”), sugieren la eliminación de los mismos, por la ambigüedad factorial que presentan, ya que el primero satura con el factor estilos alimenticios y externalidad, pero también se vincula con el reactivo 24 del factor actividad física programada; y el segundo satura en el factor actividad física cotidiana, pero se relaciona con los ítems 36 ("poner más esfuerzo y hacer más abdominales y pesas") y 24 ("realizar rutinas de ejercicios a pesar de que no tengas ganas de hacerlas") de actividad física programada. Sin 
embargo, cuando se intentó eliminarlos no se mejoró el ajuste del modelo confirmatorio. Debe señalarse, entonces, que la condición de estos ítems representa una limitación del instrumento de mujeres que no se resuelve ni con la eliminación de los mismos, lo que sugiere agregar y estudiar nuevos, cuyos análisis arrojen definición factorial.

En cuanto a las aportaciones de este estudio, debe enfatizarse la contribución teórico-práctica relacionada con la disponibilidad de instrumentos válidos y confiables, ajustados a las necesidades y género de escolares mexicanos.

Algunas de las implicaciones prácticas del $A P-C P$ plantean la posibilidad de diagnosticar a niños y adolescentes en cuanto a la autovaloración de autoeficacia para realizar actividad física y control de peso; $y$, por lo tanto, incidir en la prevención del problema de obesidad que aqueja a la población mundial, particularmente a los mexicanos en las primeras etapas de vida.

\section{REFERENCIAS}

Acosta García, M. V., Llopis Marín, J. M., Gómez Peresmitré, G. \& Pineda García, G. (2005). Estudio transcultural entre adolescentes de España y México. International Journal of Psychological Therapy, 5(3), 223-232.

Aedo, A. \& Ávila, H. (2009). Nuevo cuestionario para evaluar la autoeficacia hacia la actividad física en niños. Revista Panamericana de Salud Pública, 26(4), 324-329.

Alvariñas Villaverde, M., Fernandez Villarino, M. A. \& López Villar (2009). Actividad física y percepciones sobre deporte y género. Revista de Investigación en Educación, 6, 113-122.

American Diabetes Association. (2010). Chronic Gum Disease may lead to diabetes. Recuperado el 13 de Septiembrede2010dehttp://www.diabetes.org/newsresearch/research/access-diabetes-research/chronicgum-disease-may-lead.html

Andersen, R. E., Wadden, T. A., Bartlett, S. J., Zemel, B., Verde, T. J. \& Franckowiak, S. C. (1999). Effects of lifestyle activity vs. structured aerobic exercise in obese women: a randomized trial. JAMA, 281(4), 335-340.
Para finalizar, se hace hincapié en las bondades del uso del análisis factorial confirmatorio: a) al adaptar instrumentos ya desarrollados se conoce de antemano qué ítems deben medir qué dimensiones, por lo que dicho análisis corrige las deficiencias inherentes a la perspectiva exploratoria y conduce a una mayor concreción de las hipótesis que deben ser contrastadas; $b$ ) la mayoría de las veces el diseño de un instrumento no consiste solamente en un grupo de ítems relativos al constructo global que se está estudiando, sino en subconjuntos de ítems específicos para cada factor, por lo que la asignación de indicadores específicos a dimensiones concretas es una de las mayores aportaciones de la perspectiva confirmatoria; $c$ ) los modelos generados en el análisis factorial confirmatorio permiten contrastar la validez ajustando un modelo que la asuma y diagnosticando su bondad de ajuste (validación de constructo).

Armenta González-Palenzuela, F. (2004). La motivación y adhesión hacia la actividad física y el deporte. Escuela Abierta, 7, 137-152.

Astudillo García, C. \& Rojas Russel, M. (2006). Autoeficacia y disposición al cambio para la realización de actividad física en estudiantes universitarios. Acta Colombiana de Psicología, 9(001), 41-49.

Bandura, A. (1998). Self-efficacy, the Exercise of Control. New York: WH Freeman.

Batty, D. \& Lee, I-Min. (2004). Physical activity and coronary heart disease. $B M J, 328,1089$.

Bollen, K .A. \& Long, J. S. (1993). Testing structural equation models. CA: Sage.

Bussey, K. \& Bandura, A. (1999). Social Cognitive Theory of Gender Development and Differentiation. Psychological Review, 106, 676-713.

Cabrera, G., Gómez, L. \& Mateus, J. C. (2004). AF y etapas del cambio comportamental en Bogotá. Colombia Médica, 35(2), 82-86.

Clark, M. M., Abrams, D. B., Niaura, R. S., Eaton, C. A. \& Rossi, J. S. (1991). Self-efficacy in weight management. Journal of Consulting and Clinical Psychology, 59(5), 739-744. 
Daniels, S. R. \& Greer, F. R. (2008). Committee on Nutrition. Lipid Screening and Cardiovascular Health in Childhood. Pediatrics, 122(1), 198-208.

Dunn, A., Marcus, B., Kampert, J., Garcia, M., Kohl, H. \& Blair, S. (1999). Comparison of lifestyle and structured intervention to increase pshysical activity and cardiorespiratory fitness. A randomized trial. JAMA, 27(4),327-334.

Flórez Alarcón, L. (2007). Psicología Social y de la Salud. Promoción y Prevención. Colombia: Manual Moderno.

Flores León, A., González-Celis Rangel, A. L. \& Valencia Ortiz, A. (2010). Validación del Instrumento de Autoeficacia para Realizar Conductas Saludables en Niños Mexicanos Sanos. Revista Psicología y Salud, 20(1), 23-30.

Gila, A., Castro, J., Toro, J. \& Salamero, M. (2004). Subjective body image dimensions in normal female population: evolution trough adolescence and early adulthood. International Journal of Psychology and Psychological Therapy, 4, 1-10.

Glynn, S.M. \& Ruderman, A.J.(1986). The development and validation of an eating self-efficacy scale. Cognitive Therapy and Research, 10(4), 403-420.

Hair, J. E., Anderson, R. E., Tatham, R. L., \& Black W. C. (1998). Multivariate data analysis. (5th ed.). Upper Saddle River, NJ: Prentice-Hall.

Jago, R., Baranowski, T., Baranowski, J. C., Cullen, K. W. \& Thompson, D. (2007). Distance to food stores \& adolescent male fruit and vegetable consumption: mediation effects. International Journal of Behavioral Nutrition Physical Activity, 13, 4(1), 1-14.

Juarbe, T., Lipson, J., \& Turok, X. (2003). Physical Activity Beliefs, Behaviours and Cardiovascular Fitness of Mexican Immigrant Women. Journal Transcultural Nursing, 14(2)108-116.

Kuhl, J. (1994). A theory of action and state orientations. En J, Kuhl \& J. Beckman (Eds.). Volition an personality: Action versus state orientation (pp. 47-59). Gottingen: Hogrefe.

León Hernández, R., Gómez-Peresmitré, G. \& Platas Acevedo, S. (2008). Conductas alimentarias de riesgo y habilidades sociales en una muestra de adolescentes mexicanas. Salud Mental, 31, 447-452.

Lomax, G. R. (1982). A guide to LISREL-type structural equation modeling. Behavior Research Methods \& Instrumentation, 14, 1-8.
Lorig, K., Stewart, A., Ritter, P., Gonzalez, V., Laurent, D. \& Lynch, J. (1996). Outcome Measures for Health Education and Other Health Care Interventions. Thousand Oaks, CA: SAGE Publications.

Márquez, D. X. \& MacAuley, E. (2006). Social cognitive correlates of leissure time physical activity among Latinos. Journal of Behavioral Medicine, 29(3), 281289.

Pianosi, P. \& Davis, H. (2004). Determinants of Physical Fitness in Children with Asthma. Pediatrics, 113(3), 225-229.

Povey, R., Conner, M., Sparks, P., James, R. \& Shepherd, R. (2000). Application of the therapy behaviour theory to two dietary behaviours: Roles of perceived control and self-efficacy. British Journal of Health Psychology, 5(2), 21-139.

Román, Y., Díaz, B., Cárdenas, M., \& Lugli, Z. (2007). Construcción yvalidación del Inventario Autoeficacia Percibida para el Control de Peso. Clínica y Salud, 18, 1, (abril-mayo). España: Madrid.

Ruiz, V. M., Berrocal, C., López, A. E. \& Rivas, T. (2002). Instrumentos de evaluación de la autoeficacia percibida en el control de conductas de ingesta. Propiedades Psicométricas. Trabajo presentado en el V Congreso de Evaluación. Málaga, España.

Ruiz, V. M., Berrocal, C., López, A. E. \& Rivas, T. (2003). Autoeficacia en el control de la ingesta. Adaptación al castellano de la Eating Self Efficacy Scale. Psicothema, 15 (001), 36-40

Ryan, M. P. (2005). Physical activity levels in Young adult hispanics and whites. Social Cognitive Determinants, 20(6), 709-727.

Saldaña, C. (1994). Trastornos del comportamiento alimentario. Madrid: Fundación Universidad-Empresa.

Saldaña, C. \& Rossell, R. (1988). Obesidad. Barcelona: Martínez Roca.

Salmon, J. \& Timperio, A. (2007). Prevalence Trends and Environmental Influences on Child and Youth Physical Activity. Medicine Sport Science, 50, 183 199.

Stice,E., Shaw, H., \& Marti, N. A. (2006). Meta-Analytic Review of Obesity Prevention Programs for Children and Adolescents: The Skinny on Interventions That Work. Psychological Bulletin, 132 (5), 667-691.

Schwarzer, R. \& Jerusalem, M. (1995) Generalized SelfEfficacy Scale. En J. Weinman, S. Wright \& $\mathrm{M}$. 
Johnson (eds.) Measures in health psychology: A user's portfolio, Causal and control beliefs. (pp. 35-37). Windsor England: NFER-NELSON.

Schwarzer, R. \& Renner, B. (2000). Social-cognitive predictors of health behavior. Action self-efficacy and coping self-efficacy. Health Psychology, 19, 487-495.

Tercedor Sánchez, P. (2001). Actividad física, condición física y salud. Sevilla: Wanceulen.
Vinaccia, S., Contreras, F., Restrepo Londoño, L. M., Cadena, J. \& Anaya, J.M. (2005). Autoeficacia, desesperanza aprendida e incapacidad funcional en pacientes con diagnóstico de artritis reumatoide. International Journal of Clinical and Health Psycho $\log y, 5,129-142$.

Weinberg, R. (1966). Fundamentos de la psicología del deporte y el ejercicio. Barcelona: Ariel Psicología. 